\title{
Radiotherapy for Hidradenitis Suppurativa: A Systematic Review
}

\author{
Alyssa M. Thompson ${ }^{a}$ Justine Seivright ${ }^{b}$ Swetha Atluria ${ }^{a}$ Amanda Ederle ${ }^{c}$ \\ Joanna Jaros ${ }^{d}$ Jennifer L. Hsiao ${ }^{\text {e Vivian Y. Shi }}{ }^{f}$ \\ ${ }^{a}$ College of Medicine, University of Arizona, Tucson, AZ, USA; ${ }^{b}$ David Geffen School of Medicine, University of \\ California, Los Angeles, CA, USA; 'C College of Medicine, University of Arkansas for Medical Sciences, Little Rock, AR, \\ USA; 'Division of Dermatology, Cook County Hospitals and Health System, Chicago, IL, USA; ' Department of Medicine, \\ Division of Dermatology, University of California Los Angeles, Los Angeles, CA, USA; ${ }^{f}$ Department of Dermatology, \\ University of Arkansas for Medical Sciences, Little Rock, AR, USA
}

\section{Keywords}

Radiotherapy · X-ray · Hidradenitis suppurativa · Therapy ·

Radiation

\begin{abstract}
Background: Hidradenitis suppurativa (HS) is a chronic inflammatory dermatosis characterized by painful nodules, abscesses, sinus tracts, and scarring mainly in the intertriginous areas. Patients with HS often experience inadequate responses to traditional treatment consisting of lifestyle modification, topical and systemic antibiotics, hormonal modulators, biologics, and procedural modalities. Low-dose radiotherapy has been used in benign cutaneous conditions, including HS; however, there is a paucity of literature summarizing its evidence. Herein, we systematically review the current literature on the efficacy of radiotherapy for patients with HS. Summary: This systematic review of the published literature reports the patient demographics, treatment regimens, efficacy, and adverse effects of radiotherapy in the treatment of HS. The historic timeline of these publications highlights the changes in management recommendations, introduction of more standardized outcome measures, and enhancements in treatment options. Radiotherapy appears
\end{abstract}

to be an option for patients with treatment-resistant HS or who are poor surgical candidates. However, there remains a paucity of consensus on proper candidate selection, dosing, efficacy, and safety of the short- and long-term effects of radiotherapy.

(c) 2021 S. Karger AG, Basel

\section{Introduction}

Hidradenitis suppurativa (HS) is a chronic inflammatory dermatosis characterized by painful nodules, abscesses, sinus tracts, and scarring mainly in the intertriginous areas. Current widely accepted treatment options for HS include various combinations of lifestyle modifications, topical and systemic antibiotics, hormonal modulators, biologics, and procedural modalities. However, the aforementioned treatments often lead to inadequate response even when used in a combined fashion, especially in moderate and severe HS patients. Additionally, these treatment options may be contraindicated in some patients due to drug interactions, side effects, comorbidities, or poor surgical candidacy. karger@karger.com

www.karger.com/drm

(c) 2021 S. Karger AG, Basel

Karger ${ }^{\prime}=$
Vivian Y. Shi

Department of Dermatology

University of Arkansas for Medical Sciences

4301 W. Markham St., 576, Little Rock, AR 72205-7199 (USA)

vivian.shi.publications@gmail.com 
Radiation treatment, also known by terms such as radiation therapy, radiotherapy, irradiation, or X-ray therapy, administers high-energy particles or waves to diseased areas resulting in cellular DNA damage and death [1]. For this review we will use the term radiotherapy. The millisievert $(\mathrm{mSv})$ is used to define the average accumulated radiation dose to an individual for 1 year where $1 \mathrm{mSv}$ is the dose produced by $1 \mathrm{mGy}$ of radiation [2]. High-dose (more than $250 \mathrm{mSv}$ ) radiotherapy is commonly used in malignant diseases, and low-dose radiotherapy (under $100 \mathrm{mSv}$ ) has been used to treat benign inflammatory conditions such as keloids, eczema, and psoriasis due to its anti-inflammatory and immunomodulating effects [3-6].

Nearly a century ago, small reports on radiotherapy for patients with HS were published signaling an interest in exploring this treatment modality. The North American Clinical Management Guidelines for HS currently rank external beam radiotherapy as a grade $\mathrm{C}$ recommendation (based on consensus, usual practice, opinion, disease-oriented evidence, or case series) and presents it as an option for patients with treatment-resistant HS who are poor excisional candidates $[7,8]$. Currently there is a paucity of literature summarizing evidence regarding the use of radiotherapy in HS. Herein, we systematically review the current literature on the efficacy of radiotherapy for patients with HS.

\section{Methods}

In August 2020, a systematic review was conducted by 2 independent authors (S.A. and J.S.) on PubMed and EMBASE for articles from 1950 to 2020 . The following search terms were used: ("hidradenitis suppurativa" OR "suppurative hidradenitis" OR "hidradenitis" OR "hidradenitides" OR "acne inversa” OR "velpeau disease" OR "verneuil disease") AND ("radiotherapy" OR "brachytherapies" OR "brachytherapy" OR "electro magnetic" OR "electromagnetic" OR "irradiation" OR "radiation" OR "radio therapies" OR "radio therapy" OR "radio treatment" OR "radio treatments" OR "radiotherapies" OR "radiotherapy" OR "radiotreatment" OR "radiotreatments" OR "roentgen therapies" OR "roentgen therapy" OR "roentgen treatment" OR "roentgen treatments" OR "roentgenotherapies" OR "roentgenotherapy" OR "roentgenotherapy" OR "rontgen therapies" OR "rontgen therapy" OR "rontgen treatment" OR "rontgen treatments" OR "therapeutic radiology" OR "X-ray therapies" OR "X-ray therapy" OR "X-ray treatment" OR "X-ray treatments" OR "xray therapies" OR "xray therapy" OR "xray treatment" OR "xray treatments"). Articles were manually screened based on title, abstract, and full text. For abstract-only publications, the full text article was found when possible. Articles were included if they were in the English language, contained original data (non-review and non-commentary), and the study population consisted of HS patients and radio- therapy for HS was discussed. Reference screening for additional relevant articles was then completed on all included articles. Of note, relevant review articles and commentaries also underwent reference screening but were not included in this review. The Preferred Reporting Items for Systematic Reviews and Meta-Analyses (PRISMA) flow diagram was used to track the search schema (Fig. 1). All radiation units were converted to gray (Gy) for direct comparison.

\section{Results}

A total of 591 citations were identified from the literature search (Fig. 1). Of these, 9 articles examined radiotherapy in HS and met inclusion criteria. Among the 9 articles reviewed, there was a total of 122 patients in the studies; 105 patients were in studies published before the year 2000 and 17 patients in studies published after 2000 . Table 1 summarizes the study design, patient demographics, interventions, prior treatments, treated anatomical sites, results, and reported side effects of the included studies.

The first report of radiotherapy in HS was in 1950. Fifty-four patients (25 male, 29 female, mean age 25 years) with either hyperacute or chronic HS of the axilla were exposed to radiotherapy 3 times a week for 5-10 treatments at a per fraction dose of $1 \mathrm{~Gy}$ for hyperacute HS and 1.5-2 Gy for chronic HS. The total radiotherapy dose for both groups was 10-12 Gy. Patients also concomitantly received incisions. Patients with a single abscess responded in an average of 3 treatments and resolution of an affected axilla took an average of 7.8 treatments. All patients responded well to treatments with no recurrences and most pain was gone by $2-3$ treatments [9].

Five years later, a case series was published with 45 patients (23 acute, 10 chronic localized, and 12 chronic generalized) who had inadequate responses to prior treatments (various topical applications, bathing additives, systemic antibiotics, incision and drainage, and surgery), and who had undergone radiotherapy of unknown dose and duration. In addition to radiotherapy, a combination of hot compresses and systemic antibiotics were used for the acute cases; patients with chronic localized cases also received an unknown dose of autogenous vaccines and staphylococcus toxoid injections. Treated sites included axilla, groin, and "generalized" regions including the mammary fold, umbilical region, and Montgomery's glands of the areola. Results varied based on the disease course; patients with acute disease had no recurrences, patients with chronic localized disease could expect re- 
Fig. 1. PRISMA flow diagram (from Moher

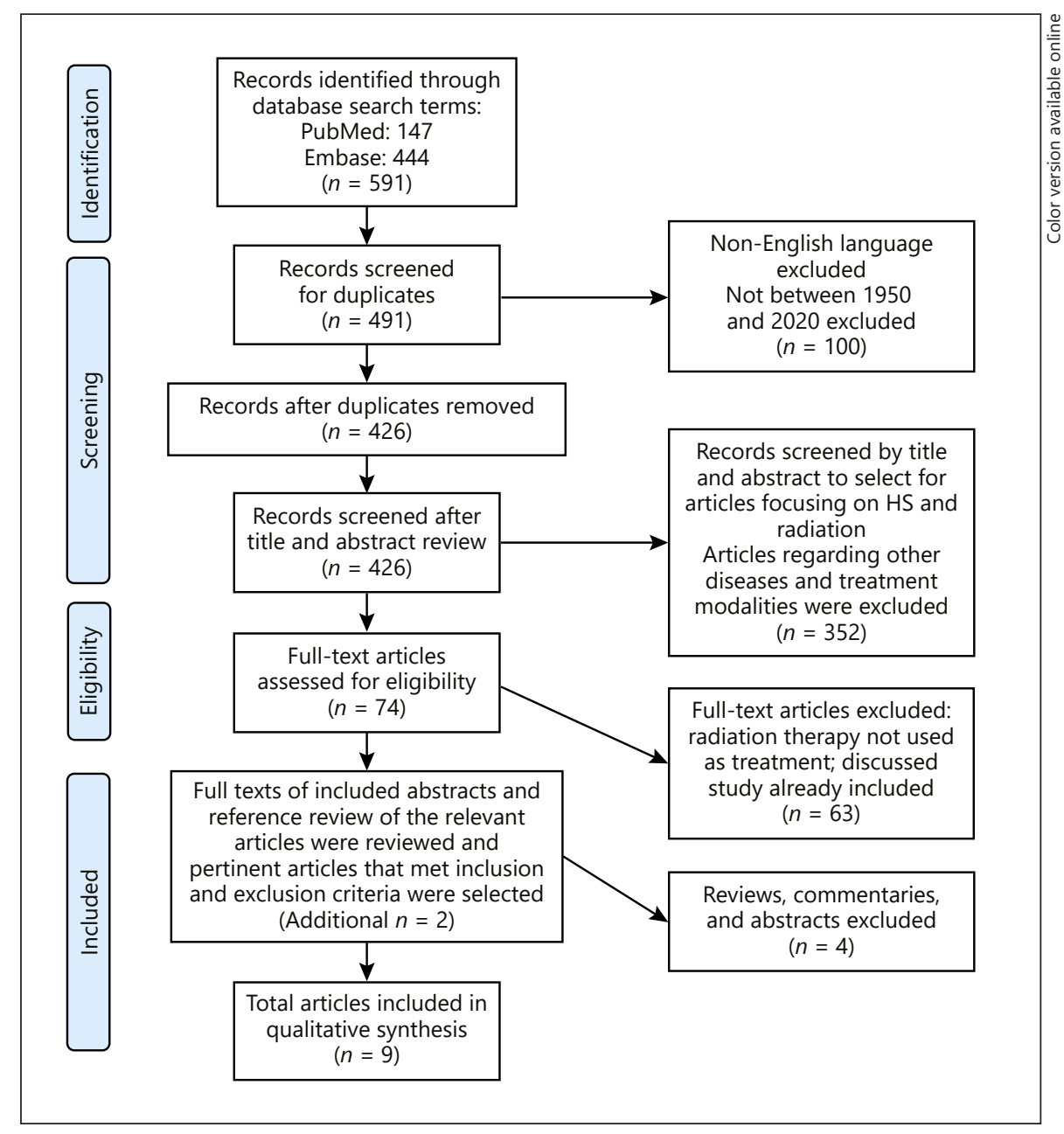
et al. [24]).

currences, and patients with chronic generalized disease had a uniformly poor response [10].

Beginning in 1965, a single course of radiotherapy at lower doses became the treatment of choice for HS. A case series described 5 patients aged $22-51$ years who were exposed to a single course of 4.5 Gy to the affected axilla. Within a few weeks of treatment, most patients only had a few active lesions. There was no mention of supplementary therapy in congruence with radiotherapy. No active lesions in treated sites were reported at follow-up periods ranging from months to years after treatment [11].

After the 1965 case series, there was a halt in publications on HS treated with radiotherapy for nearly 30 years. The reason for this is unknown, however the historical timeline of radiation discoveries and safety in the USA may provide insight. Prior to 1945 , radiation safety concerns centered around those with occupational exposures. After the 1945 bombing of Hiroshima, radiation exposure became a public risk. Up until 1959, private or-

Radiotherapy for Hidradenitis Suppurativa: A Systematic Review ganizations were in charge of determining radiation safety standards in the USA. In 1964 the National Committee on Radiation Protection and Measurements (NCRP) was granted a federal charter through the US congress which enacted as Public Law to collect and inform the public on radiation protection, likely provoking concerns of the potential health risk of radiation exposure. From this point in time to the present day, newer, more refined studies on radiation risks and recommendations have been published, likely prompting the resurgence of HS radiotherapy literature [12].

After nearly a 3-decade cessation, literature on this topic reemerged in the mid-1990s, with the majority of the articles published in the 2010s. Radiotherapy was used on axillary HS in a 29-year-old male with AIDS. Several days after a single dose of $4 \mathrm{~Gy}$, the suppuration and pain associated with his HS resolved. Unfortunately, 2 months after treatment the patient died due to unreported causes [13]. 


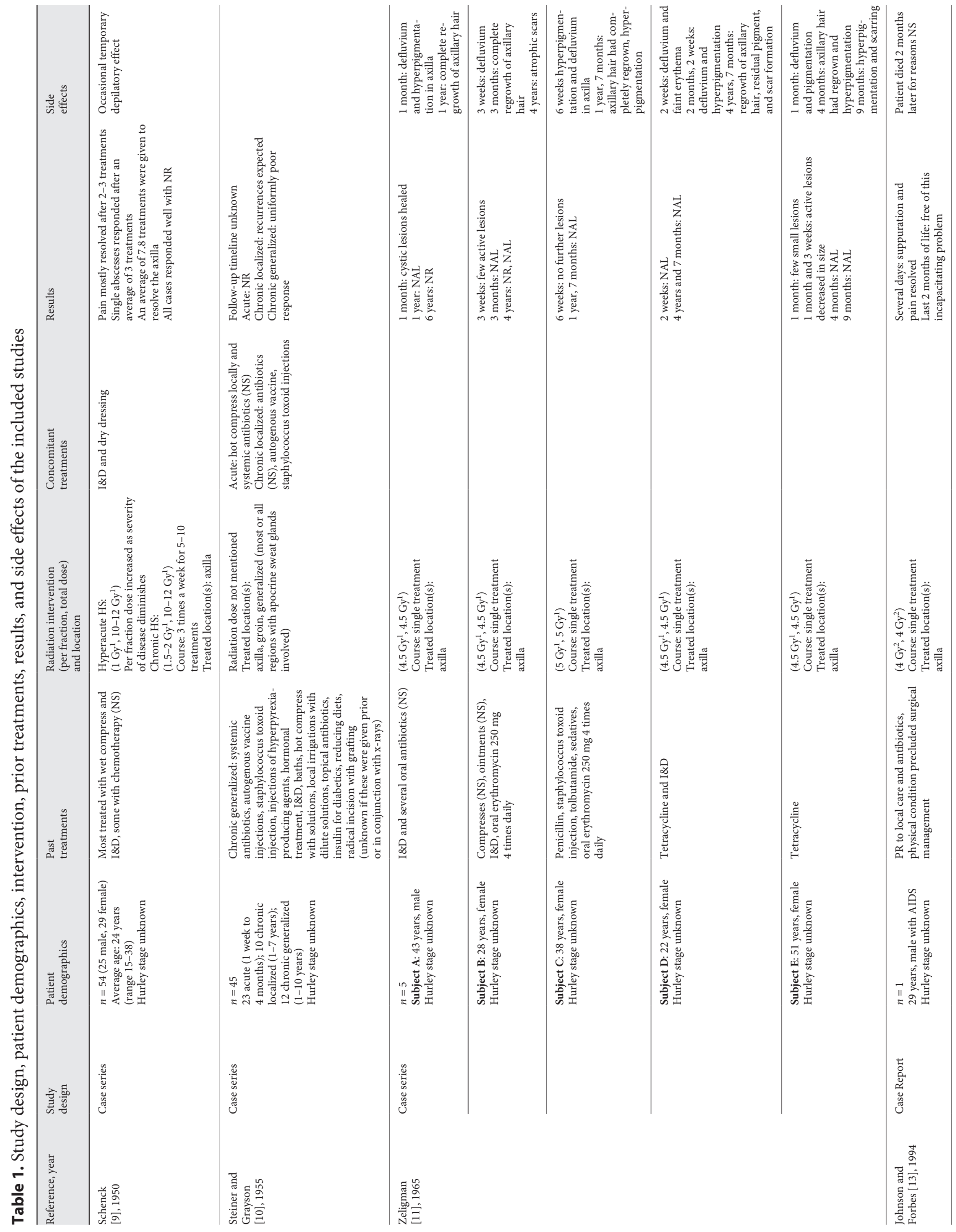




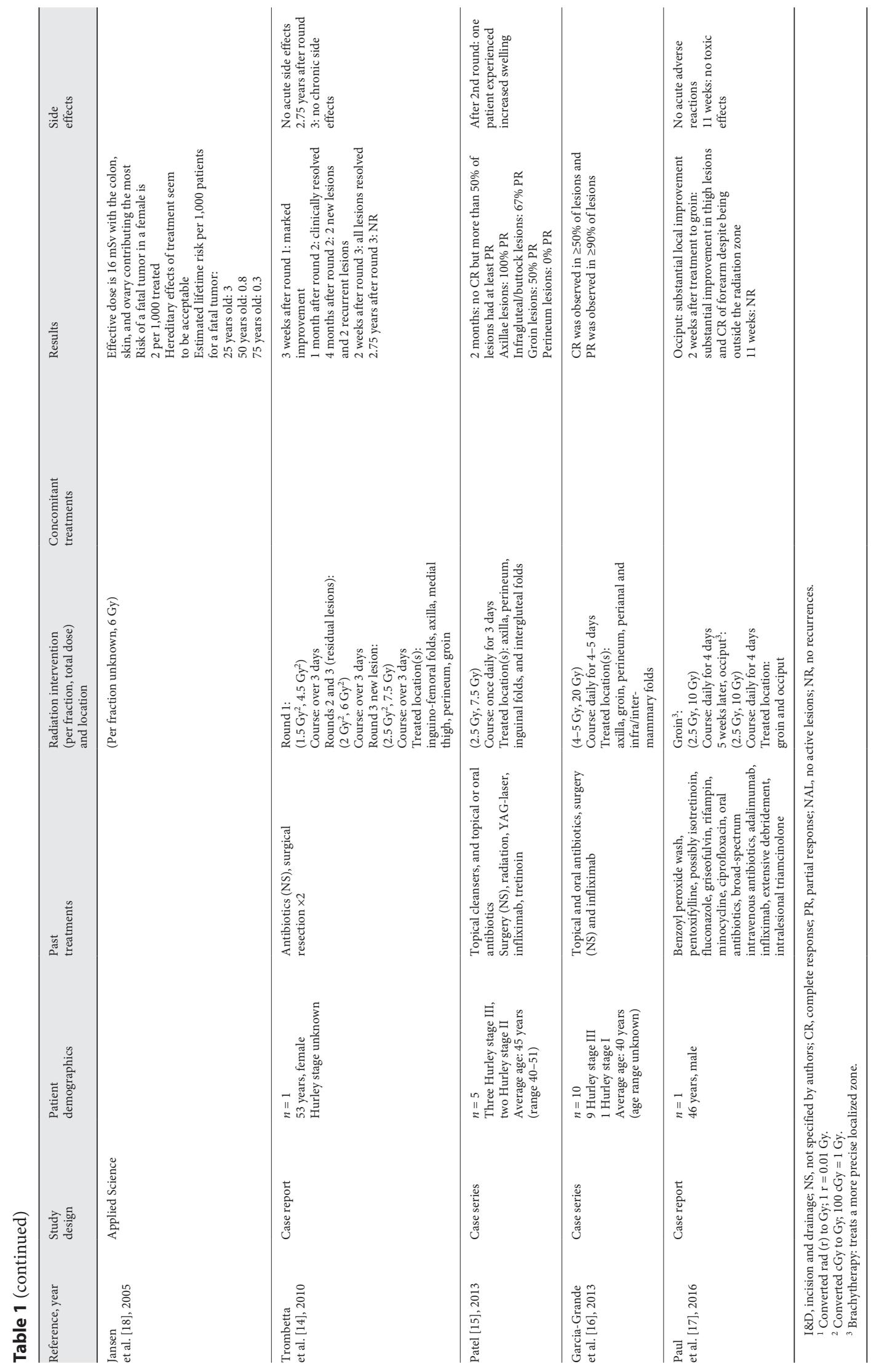


Prior to 2010, the radiotherapy dose patients received was determined at the beginning of treatment and not adjusted. In 2010 a case report on a 53-year-old female was presented introducing a new radiotherapy time course. This time course incorporated multiple rounds of treatment at various fractions and total doses depending on the stage of the lesion. All rounds of radiotherapy were received over the course of 3 days. During round 1, the patient received a total of 4.5 Gy at 1.5 per fraction. During round 2 , the residual lesions were treated at a total dose of 6 Gy at 2 Gy per fraction. This protocol resulted in clinical resolution a month after therapy. Four months later, 2 lesions had recurred and 2 new lesions were present. The recurrent lesions received another round of radiotherapy for a total dose of 6 Gy at 2 Gy per fraction and the new lesions received a total dose of 7.5 Gy at 2.5 Gy per fraction. Two weeks later, all lesions resolved and nearly 3 years later the patient has had no recurrences [14].

In 2013, a case series reported that the response rate of HS lesions to radiotherapy appears to vary based on the anatomical location. Five patients were treated with radiotherapy at $2.5 \mathrm{~Gy}$ per fractions once daily for 3 days for a total dose of 7.5 Gy. Two months later, the authors found that at least partial improvement was observed in $100 \%$ of the axillae lesions, $67 \%$ of the infragluteal/buttocks lesions, and $50 \%$ in the groin lesions. No complete responses were observed, and there was a lack of response to radiotherapy in the perineum [15]. A second case series published in 2013 with 10 patients used a higher per fraction dose and total dose (20 Gy at 4-5 Gy) over the course of 2-5 days and found that a complete response was observed in $\geq 50 \%$ of lesions and a partial response was observed in $\geq 90 \%$ of lesions [16].

Most recently in 2016, a 45-year-old man with severe HS was reported to have been treated with a new form of radiotherapy called brachytherapy, which is used to treat a more precise localized zone. The treatment consisted of a total dose of 10 Gy at 2.5 Gy per fraction daily for 4 days to the groin and occiput. Substantial improvement was noted at both anatomical locations. Of note, lesions on the thigh and forearm also resolved despite being outside the treatment zone (abscopal effect). No recurrences were noted at 11 weeks of follow-up [17].

Among all aforementioned studies, patients had tried several other therapies prior to the initiation of radiotherapy. The most common side effects included temporary defluvium (hair loss) at treated sites, erythema, hyperpigmentation, and atrophic scaring.

In 2005, the first risk analysis of radiotherapy was performed to model the risk of fatal tumor development. The treatment framework was designed from a treatment regimen used on a female patient who received a total dose of 6 Gy for HS of the groin. The authors found that the risk of fatal tumor development in a woman was 2 per 1,000 treated, with the most at-risk organs being the colon, skin, and ovary. Of note, lifetime risk of fatal tumor development decreased with increased age at radiotherapy treatment ( 3 per 1,000 for 25 years of age, 0.8 per 1,000 for 50 years of age, and 0.3 per 1,000 for 75 years of age). Mutagenic effects of radiotherapy to the gametes are relatively low, thus radiation effects to the offspring seem to be acceptable [18].

\section{Discussion}

Radiotherapy appears to be a viable option for patients with treatment-resistant HS or who are poor surgical candidates, such as those with disease in inoperable or extensive anatomical locations, and those with specific medical comorbidities. Among the reviewed studies, patients experienced positive results with very minor adverse effects. However, long-term follow-ups were not reported so long-term adverse effects such as secondary malignancy are unknown. In general, chronic radiotherapy can be associated with more serious and permanent adverse cutaneous effects, including textural changes, cutaneous breakdown, fibrosis, poikilodermatous changes, loss of hair follicles and sweat glands, loss of nail appendages, and secondary malignancies (primary squamous cell carcinoma and basal cell carcinoma) [19]. Patients with chronic HS ulcerations have an increased risk of squamous cell carcinoma. However, it is unknown if radiotherapy will increase or decrease this risk. Decreasing the inflammation with appropriate HS treatments may reduce the risk of squamous cell carcinoma but it is possible that exposure to radiation may increase the risk due to the additive effect. Thus, HS patients should be closely monitored for cutaneous malignancy despite achieving disease remission. Quantifying the risk of secondary malignancy in each anatomical site is important in HS patients as the risk may vary by location.

Radiotherapy in HS was cited as a potential treatment option in the mid-1900s and has regained attention in more current literature after a 3-decade break. Currently, a phase I clinical trial (NCT03040804) examining the safety of radiotherapy in the treatment of advanced $\mathrm{HS}$ is in the recruitment phase; 5 fractions of 1.5 Gy will be introduced over 1 week for a total dose of $7.5 \mathrm{~Gy}$ and patients will be followed for 3 months to observe changes in 
quality of life, cutaneous discharge, and immunohistochemistry [20]. Furthermore, radiotherapy has also been used in dissecting cellulitis and may be a therapeutic option for patients with multiple follicular occlusion diseases by targeting multiple anatomic locations [21]. Future research is needed to determine the mechanism of action of radiotherapy in the treatment of HS as well as the optimal dose and duration of use. In addition, larger studies to allow for subgroup analysis of treatment efficacy based on lesion location, lesion type, disease severity, and patient demographics can aid clinicians in choosing optimal candidates for this treatment modality. Finally, long-term safety data is needed, as well as monitoring protocols for cutaneous malignancy at treatment sites. Limitations of the reviewed literature include lack of standard reporting of disease and symptom severity, dynamic disease course, lesion types, and outcome measures, thus creating challenges for comparisons across reports.

\section{Conclusion}

Recent literature has highlighted the potential benefits of radiotherapy in HS. Future clinical investigations are needed to guide candidate selection and establish the dosing, efficacy, and safety of short- and long-term effects of radiotherapy. Incorporating newly developed clinical and patient-oriented grading tools can be important for standard interpretation of efficacy $[22,23]$. With improved understanding of the aforementioned factors, radiother- apy can be strategically incorporated to complement other medical, procedural, and lifestyle interventions used in HS management, particularly in patients that are poor surgical candidates.

\section{Key Message}

Cumulative literature has highlighted the potential benefits of radiotherapy in hidradenitis suppurativa. Radiotherapy may be considered for patients with treatment-resistant hidradenitis suppurativa or those who are poor surgical candidates. Future investigations are needed to determine the optimal radiotherapy regimen and strategies to incorporate it into current management plans.

\section{Conflict of Interest Statement}

V.Y.S. is on the board of directors for the Hidradenitis Suppurativa Foundation (HSF), a stock shareholder of Learn Health, and has served as an advisor, investigator and/or speaker for Sanofi Genzyme, Regeneron, AbbVie, Burt's Bees, Dermira, Eli Lilly, Novartis, Pfizer, Galderma, Leo Pharma, SUN Pharma, Menlo Therapeutics, GpSkin, and Skin Actives Scientific. J.L.H. has served as an advisor for Novartis.

\section{Funding Sources}

There was no funding for this study.

\section{Author Contributions}

All authors met the ICMJE criteria for authorship.

\section{References}

1 Toulany M. Targeting DNA double-strand break repair pathways to improve radiotherapy response. Genes. 2019 Jan;10(1):25.

2 Carr S. The millisievert and milligray as measures of radiation dose and exposure [Internet]. Available from: https://www.mun.ca/biology/scarr/Radiation_definitions.html.

3 Arenas M, Sabater S, Hernández V, Rovirosa A, Lara PC, Biete A, et al. Anti-inflammatory effects of low-dose radiotherapy. Indications, dose, and radiobiological mechanisms involved. Strahlenther Onkol. 2012 Nov; 188(11):975-81.

4 Yang X, Shao Y, Yu W, Zhang X, Sun Y, Zhang L, et al. A Novel Radiotherapy Approach for Keloids with Intrabeam. BioMed Res Int. 2019 Jul;2019:4693528.

5 Sumila M, Notter M, Itin P, Bodis S, Gruber $\mathrm{G}$. Long-term results of radiotherapy in patients with chronic palmo-plantar eczema or psoriasis. Strahlenther Onkol. 2008 Apr; 184(4):218-23.
6 Fleischmann D. Radiation Dose and Radiation Risk [Internet]. Department of Radiology Stanford University. Available from: https://med.stanford.edu/content/dam/sm/ cvimaging/documents/lectures/18DEC13 Fleischmann_RadiationDoseRisk_final_ HANDOUT.pdf.

7 Alikhan A, Sayed C, Alavi A, Alhusayen R, Brassard A, Burkhart C, et al. North American clinical management guidelines for hidradenitis suppurativa: A publication from the United States and Canadian Hidradenitis Suppurativa Foundations: Part I: Diagnosis, evaluation, and the use of complementary and procedural management. J Am Acad Dermatol. 2019 Jul;81(1):76-90.

8 Ebell MH, Siwek J, Weiss BD, Woolf SH, Susman J, Ewigman B, et al. Strength of recommendation taxonomy (SORT): a patient-centered approach to grading evidence in the medical literature. J Am Board Fam Pract. 2004 Jan-Feb;17(1):59-67.
9 Schenck SG. Hidradenitis suppurativa axillaris; an analysis of 54 cases treated with roentgen rays. Radiology. 1950 Jan;54(1):747.

10 Steiner K, Grayson LD. Hidradenitis suppurativa of the adult and its management. AMA Arch Derm. 1955 Feb;71(2):205-11.

11 Zeligman I. Temporary $\mathrm{x}$-ray epilation therapy of chronic axillary hidradenitis suppurativa. Arch Dermatol. 1965 Dec;92(6):690-4.

12 Jones CG. A review of the history of U.S. radiation protection regulations, recommendations, and standards. Health Phys. 2005 Jun; 88(6):697-716.

13 Johnson SA Jr, Forbes RC. Hidradenitis in a patient with AIDS: palliation with superficial radiation. South Med J. 1994 Apr;87(4):549.

14 Trombetta M, Werts ED, Parda D. The role of radiotherapy in the treatment of hidradenitis suppurativa: case report and review of the literature. Dermatol Online J. 2010 Feb;16(2): 16. 
15 Patel SH. Radiation Therapy for Chronic Hidradenitis Suppurativa. J Nucl Med Radiat Ther. 2013;4:146

16 Garcia-Grande A, Herranz Pinto P, Diaz Diaz R. Radiation therapy for refractory hidradenitis suppurativa. Rep Pract Oncol Radiother. 2013 Jun; 18:S358.

17 Paul S, Bach D, LeBoeuf NR, Devlin PM, Lipworth AD. Successful use of brachytherapy for a severe hidradenitis suppurativa variant. Dermatol Ther. 2016 Nov;29(6):455-8.

18 Jansen JT, Broerse JJ, Zoetelief J, Klein C, Seegenschmiedt HM. Estimation of the carcinogenic risk of radiotherapy of benign diseases from shoulder to heel. Radiother Oncol. 2005 Sep;76(3):270-7.
19 Bray FN, Simmons BJ, Wolfson AH, Nouri K. Acute and Chronic Cutaneous Reactions to Ionizing Radiation Therapy. Dermatol Ther. 2016 Jun;6(2):185-206.

20 Low Dose Radiotherapy for Advanced Hidradenitis Suppurativa (RTHS) [Internet]. ClinicalTrials.gov. Available from: https:// clinicaltrials.gov/ct2/show/NCT03040804?te $\mathrm{rm}=\mathrm{NCT} 03040804 \&$ draw $=2 \& \mathrm{rank}=1$.

21 Chinnaiyan P, Tena LB, Brenner MJ, Welsh JS. Modern external beam radiation therapy for refractory dissecting cellulitis of the scalp. Br J Dermatol. 2005 Apr;152(4):777-9.

22 Finlay AY, Khan GK. Dermatology Life Quality Index (DLQI) - a simple practical measure for routine clinical use. Clin Exp Dermatol. 1994 May;19(3):210-6.
23 Zouboulis CC, Tzellos T, Kyrgidis A, Jemec GB, Bechara FG, Giamarellos-Bourboulis EJ, et al.; European Hidradenitis Suppurativa Foundation Investigator Group. Development and validation of the International Hidradenitis Suppurativa Severity Score System (IHS4), a novel dynamic scoring system to assess HS severity. Br J Dermatol. 2017 Nov; 177(5):1401-9.

24 Moher D, Liberati A, Tetzlaff J, Altman DG. Preferred reporting items for systematic reviews and meta-analyses: the PRISMA statement. PLoS Med. 2009 Jul 21;6(7):e1000097. 